\title{
Retrieval of vertical profiles of atmospheric refraction angles by inversion of optical dilution measurements
}

\author{
D. Fussen, C. Tétard, E. Dekemper, D. Pieroux, N. Mateshvili, F. Vanhellemont, G. Franssens, and P. Demoulin \\ Belgian Institute for Space Aeronomy, 3 Avenue Circulaire, 1180 Brussels, Belgium \\ Correspondence to: D. Fussen (didier.fussen@aeronomie.be)
}

Received: 22 January 2015 - Published in Atmos. Meas. Tech. Discuss.: 2 April 2015

Revised: 18 June 2015 - Accepted: 23 July 2015 - Published: 5 August 2015

\begin{abstract}
In this paper, we consider occultations of celestial bodies through the atmospheric limb from low Earth orbit satellites and we show how the usual change of tangent altitude associated with atmospheric refraction is inseparably connected to a variation of the observed apparent intensity, for extended and pointlike sources. We demonstrate, in the regime of weak refraction angles, that atmospheric optical dilution and image deformation are strictly concomitant. The approach leads to the integration of a simple differential equation related to the observed transmittance in the absence of other absorbing molecules along the optical path. The algorithm does not rely on the absolute knowledge of the radiometer pointing angle that is related to the accurate knowledge of the satellite attitude. We successfully applied the proposed method to the measurements performed by two past occultation experiments: GOMOS for stellar and ORA for solar occultations. The developed algorithm (named ARID) will be applied to the imaging of solar occultations in a forthcoming pico-satellite mission.
\end{abstract}

\section{Introduction}

In the terrestrial and planetary atmospheres, electromagnetic waves generally do not propagate along straight lines due to refractivity gradients caused by the vertical variation of the molecular concentration. In solar, stellar, planetary and GPS radio occultations, an orbiting spectroradiometer in a low Earth orbit (LEO) measures the atmospheric transmittance as a function of the tangent altitude of the line of sight. Such measurement techniques offer a precious advantage: they are self-calibrating because the observed signal is normalized with respect to the exo-atmospheric signal.
The effect of refraction of stellar light has been used in the past for probing planetary atmospheres (see Elliot and Olkin (1996) and reference therein). Inversion of radio occultation amplitude data was proposed by Sokolovskiy (2000) as a complementary technique to the classical phase inversion. The subject of stellar scintillations is very broad and directly related to the study of atmospheric turbulence (Wheelon, 2001, 2003). Star occultations have been the main technique used by the GOMOS instrument and allowed the reconstruction of atmospheric irregularities in air density and temperature profiles (see Kan et al. (2014) and reference therein).

The refractive optical dilution (i.e. light extinction caused by atmospheric refraction) observed during Sun occultations has been considered by Miller (1967) for a rocket flight operated by the Meteorological Office, however without presenting a solution to invert the angular integration across the solar disc. More recently, high-precision refraction measurements obtained by solar imaging were recorded by the SOFIE instrument (Gordley et al., 2009) onboard the AIM satellite. They proposed an elegant method to relate the apparent Sun image flattening to the refraction angles characterizing the tangent rays emitted from the solar disc edges.

In this paper, we focus on the exploitation of the global radiative dilution experienced by light when crossing the Earth's atmospheric layers. We present an original method to retrieve the refraction angle profile from the integration of a simple differential equation, defining the ARID algorithm (Atmospheric Refraction by Inversion of Dilution). In the recent past, our team (Dekemper et al., 2013) published a method dedicated to pressure profile retrievals by analysis of Sun refraction based on the use of Zernike polynomials. In the same sense, the ARID method relies only on the first Zernike moment (i.e. the total intensity); thus it can also be 
used for pointlike sources as stars or GPS satellites. Furthermore, the refraction profile is integrated downward from the highest considered altitude, allowing the sounding of the upper atmospheric layers where refraction is very weak.

In the first section, we recall the elementary principles of atmospheric refraction and we simplify the geometrical problem by defining the useful phase screen approximation. In the second section, we derive several interrelated effects of atmospheric refraction and we show the concomitance of image flattening, shift of the apparent tangent altitude and dilution of the incoming irradiance for LEO satellites. We finally derive the differential equation subtending the ARID model. In the third section, we apply the developed algorithm to the exploitation of the transmittance data observed by the GOMOS fast photometer at $672 \mathrm{~nm}$. In Sect. 4, we revisit the 22-yearold solar occultation data recorded by the ORA instrument, and we demonstrate the possibility of directly using the Sun transmittance to retrieve the refraction angle profile, although the problem is more complicated due to the angular extension of the solar disc. In the last section we draw conclusions on the capacity of the proposed algorithm and the associated requirements.

We deliberately do not consider electromagnetic scintillation and fast amplitude fluctuations superimposed on signals that travel through a turbulent atmosphere and that can be measured by using fast photometers (Wheelon, 2001). Instead we will concentrate on the low-frequency signal extinction caused by refractive dilution. In order to limit the scope of this study, we focus on the retrieval of the profiles of refracted angles. The vertical inversion techniques coupled to the use of the hydrostatic hypothesis have been extensively studied elsewhere (Hajj et al., 2002).

\section{Atmospheric refraction and phase screen approximation}

Assuming that the Fermat's principle can be used to describe the atmospheric propagation of electromagnetic radiation in the optical domain (Born and Wolf, 1950), it can be shown that the position vector $\vec{r}$ of a light ray obeys the following equation with respect to the infinitesimal length element $\mathrm{d} s$ :

$$
\frac{\mathrm{d}}{\mathrm{d} s}\left(n(r) \frac{d \overrightarrow{\boldsymbol{r}}}{\mathrm{d} s}\right)=\vec{\nabla} n(r),
$$

where the Cartesian reference frame is located at the centre of a sphere that locally approximates the Earth geoid. If we consider the realistic case of a spherically symmetric atmosphere around the tangent point, the refractive index $n(r)$ possesses the same radial symmetry such that gradients only exist along the local vertical direction. Consequently, the ray trajectories belong to a plane defined by the light source, the Earth's centre and the receiver. This implies the existence of a conservation law similar to the angular momentum conservation for particles moving under the action of a central force:

$n\left(r_{\mathrm{t}}\right) r_{\mathrm{t}}=b$,

where $r_{\mathrm{t}}$ is the radial distance to the point of closest approach or turning point and $b$ is the impact parameter of the emitted light ray. The ray trajectory is clearly symmetrical with respect to the turning point and the total refraction angle $\alpha$, defined with respect to the incident direction, can be calculated as

$\alpha=2 \int_{r_{\mathrm{t}}}^{\infty} \frac{\mathrm{d} \alpha}{\mathrm{d} r} \mathrm{~d} r=2 \int_{r_{\mathrm{t}}}^{\infty} \frac{1}{n(r)} \frac{\mathrm{d} n(r)}{\mathrm{d} r} \frac{b}{\sqrt{n(r)^{2} r^{2}-b^{2}}} \mathrm{~d} r$.

It is important to realize that the most important contribution to $\alpha$ comes from the region around the turning point $r_{\mathrm{t}}$, i.e. at low grazing altitudes. Notice that rays are bent toward the higher-density regions implying that $\alpha \leq 0$. If we define $u=$ $n(r) r$ and $f(b)=-\alpha / 2 \pi b$, we can identify the inverse Abel transform (Bracewell, 1965) of the refractive index:

$f(b)=-\frac{1}{\pi} \int_{b}^{\infty} \frac{\ln ^{\prime}(n(u))}{\sqrt{u^{2}-b^{2}}} \mathrm{~d} u$.

It is therefore possible to retrieve a vertical profile of refractive index by using the inverse transform:

$n(u)=\exp \left(-\frac{1}{\pi} \int_{u}^{\infty} \frac{\alpha(b)}{\sqrt{b^{2}-u^{2}}} \mathrm{~d} b\right)$.

The refractive index $n(r)$ is in good approximation related to the refractivity $v(r)$ by

$n(r)=1+v(r)=1+C(\lambda) \frac{\rho(r)}{\rho_{0}}$,

where $\rho_{0}$ is the reference atmospheric density in standard conditions. A slight wavelength dependence exists for $C(\lambda)$ according to the following parameterization (Edlen, 1966):

$$
\begin{aligned}
C(\lambda)=10^{-8} & \left(8342.13+\left(2406030 . /\left(130-\left(1 . / \lambda^{2}\right)\right)\right)\right. \\
& \left.+15997 . /\left(38.9-\left(1 . / \lambda^{2}\right)\right)\right),
\end{aligned}
$$

where $\lambda$ is expressed in micrometres. It is a good approximation to consider a locally isothermal exponential atmosphere. With $H_{\mathrm{t}}$ the atmospheric scale height at the turning point (which is in turn a function of the local temperature), the refractivity is given by

$v(r) \simeq v_{\mathrm{t}} \exp \left(-\frac{r-r_{\mathrm{t}}}{H_{\mathrm{t}}}\right)$.

Keeping in mind that the refractivity $v_{\mathrm{t}}$ is a small quantity and that the ratio $r_{\mathrm{t}} / H_{\mathrm{t}}$ is large, we obtain the simple analytical approximation for $\alpha$ :

$\alpha \simeq-v_{\mathrm{t}} \sqrt{\frac{2 \pi r_{\mathrm{t}}}{H_{\mathrm{t}}}}$, 
Table 1. Refraction parameters for a standard atmosphere: $z_{\mathrm{t}}, T_{\mathrm{t}}, H_{\mathrm{t}}$ and $v_{\mathrm{t}}$ respectively refer to local tangent altitude, temperature, scale height and refractivity; $b$ is the impact parameter, $L$ the distance to the limb (satellite to phase screen), $h$ is the unrefracted impact parameter; $\alpha_{\mathrm{a}}, D_{\mathrm{a}}, \alpha_{\mathrm{b}}$ and $D_{\mathrm{b}}$ are the refraction angles and the dilution factors computed by means of formula Eq. (9) (a) or by numerical ray tracing (b).

\begin{tabular}{lllllllllll}
\hline$z_{\mathrm{t}}[\mathrm{km}]$ & $T_{\mathrm{t}}[\mathrm{K}]$ & $H_{\mathrm{t}}[\mathrm{m}]$ & $\nu_{\mathrm{t}}$ & $b[\mathrm{~km}]$ & $L[\mathrm{~km}]$ & $h[\mathrm{~km}]$ & $\alpha_{\mathrm{a}}[\mathrm{rad}]$ & $D_{\mathrm{a}}$ & $\alpha_{\mathrm{b}}[\mathrm{rad}]$ & $D_{\mathrm{b}}$ \\
\hline 0 & 288.2 & 8430.3 & $2.73 \times 10^{-4}$ & 1.7365 & 3287.8 & -60.02 & $1.88 \times 10^{-2}$ & 0.1887 & $2.56 \times 10^{-2}$ & 0.1190 \\
10 & 223.3 & 6531.9 & $9.20 \times 10^{-5}$ & 10.587 & 3270.6 & -13.00 & $7.21 \times 10^{-3}$ & 0.2463 & $5.72 \times 10^{-3}$ & 0.1881 \\
20 & 216.7 & 6338.8 & $1.98 \times 10^{-5}$ & 20.126 & 3252 & 15.01 & $1.57 \times 10^{-3}$ & 0.4645 & $1.38 \times 10^{-3}$ & 0.5264 \\
30 & 226.5 & 6625.5 & $4.10 \times 10^{-6}$ & 30.026 & 3232.4 & 28.99 & $3.19 \times 10^{-4}$ & 0.8030 & $3.37 \times 10^{-4}$ & 0.8262 \\
40 & 250.4 & 7324.6 & $8.89 \times 10^{-7}$ & 40.006 & 3212.6 & 39.79 & $6.59 \times 10^{-5}$ & 0.9535 & $8.29 \times 10^{-5}$ & 0.9515 \\
50 & 270.7 & 7918.4 & $2.29 \times 10^{-7}$ & 50.001 & 3192.6 & 49.95 & $1.63 \times 10^{-5}$ & 0.9904 & $2.04 \times 10^{-5}$ & 0.9877 \\
60 & 247 & 7225.1 & $6.88 \times 10^{-8}$ & 60 & 3172.4 & 59.98 & $5.14 \times 10^{-6}$ & 0.9977 & $5.02 \times 10^{-6}$ & 0.9970 \\
70 & 219.6 & 6423.7 & $1.84 \times 10^{-8}$ & 70 & 3152 & 70.00 & $1.46 \times 10^{-6}$ & 0.9992 & $1.24 \times 10^{-6}$ & 0.9993 \\
80 & 198.6 & 5809.4 & $4.10 \times 10^{-9}$ & 80 & 3131.5 & 80.00 & $3.42 \times 10^{-7}$ & 0.9998 & $3.08 \times 10^{-7}$ & 0.9998 \\
90 & 186.9 & 5467.1 & $7.64 \times 10^{-10}$ & 90 & 3110.8 & 90.00 & $6.58 \times 10^{-8}$ & 1.0000 & $7.64 \times 10^{-8}$ & 1.0000 \\
100 & 195.1 & 5707 & $1.27 \times 10^{-10}$ & 100 & 3090 & 100.00 & $1.07 \times 10^{-8}$ & 1.0000 & $2.04 \times 10^{-8}$ & 1.0000 \\
\hline
\end{tabular}

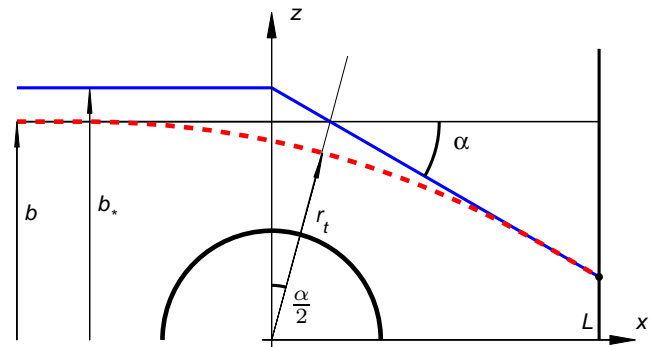

Figure 1. The phase screen approximation: the atmosphere acts as a refraction plane where the total refraction angle $\alpha$ is acquired at $x=$ 0 . For small angles, negligible errors are introduced for the impact parameter $b$ and the geolocation of the remotely sensed region.

and we give in Table 1 the values computed for a standard atmosphere. Clearly, the total refraction angle is dominated by the refractivity (hence by the density) at the turning point if the hypothesis of an exponential isothermal atmosphere holds (perturbative effects are expected at the tropopause).

It is worth noting that the effective distance $s_{\mathrm{e}}$ over which most of the refraction takes place can be estimated as the path length necessary to experience a characteristic change of $H_{\mathrm{t}}$ in the local altitude. We obtain

$s_{\mathrm{e}} \simeq \sqrt{2 r_{\mathrm{t}} H_{\mathrm{t}}} \simeq 300 \mathrm{~km}$

Considering that Eq. (3) leads to solutions with two asymptotes (straight lines before entering and after exiting the atmosphere) and that we are working in a regime of weak refraction $(|\alpha| \ll 1)$, it is legitimate to introduce the socalled "phase screen" approximation (Wheelon, 2003) that is illustrated in Fig. 1.

In the phase screen approximation, the refraction occurs through an equivalent infinitesimal atmosphere reduced to a vertical plane at the considered geolocation. The turning point position is moved horizontally by $r_{\mathrm{t}} \tan (\alpha / 2) \simeq b \alpha / 2$, in the backward direction, a negligible quantity with respect to $s_{\mathrm{e}}$. Along the vertical, the turning point is displaced up- ward by about $b \alpha^{2} / 2$ (a value smaller than the Fresnel length for $b \geq 30 \mathrm{~km}$ ), and the impact parameter $b_{*}$ can in the phase screen approximation be considered as virtually identical to $b$. Furthermore, the small magnitude of the refraction angle allows for the usual trigonometric approximation:

$\tan (\alpha) \simeq \alpha$

\section{From refraction to dilution}

Atmospheric refraction is usually considered in the context of ray tracing because the most apparent effect is a shift in the apparent tangent height of any ray emitted by a distant light source. Furthermore, in this section we are going to demonstrate that this change in position must correspond to an equivalent change in the measured spectral irradiance at the receiver.

In Fig. 2, we consider a bi-dimensional approach of the problem for an extended light source like the Sun. We also assume the following approximations: the horizontal gradients of the refractive index (i.e. of the atmospheric air density) can be neglected around the turning point, and the distance from a LEO satellite to the $\operatorname{limb}$ is about $L \simeq 3000 \mathrm{~km}$, much closer than the distance at which some refracted rays will cross the optical axis containing the centre of the Earth of radius $R$. Indeed, the maximal refraction angle $\alpha_{\mathrm{m}}$ (for a grazing ray) is about $0.02 \mathrm{rad}$ and leads to a focal length of $L_{\mathrm{m}} \simeq R / \alpha_{\mathrm{m}} \simeq 3 \times 10^{5} \mathrm{~km}$.

The angular extension of the solar disc $(\simeq 0.0093 \mathrm{rad})$ is spanned by its diameter $\Delta$ located at a distance $(L+S)$ from the satellite. Without refraction, the tangent height $h$ refers to the unrefracted ray pointing to the Sun's centre.

Any ray emitted from the Sun at altitude $z$ propagates as a straight line with slope $\beta$ until it is refracted in $x=0$ at altitude $z^{\prime}$. The exiting ray, with slope $\beta^{\prime}$, is detected at $L$ by the satellite sensor. Notice that $\beta^{\prime}$ is a priori unknown: it is necessary to use an imager with a high vertical resolution compatible with the small refraction angle, but the satellite 


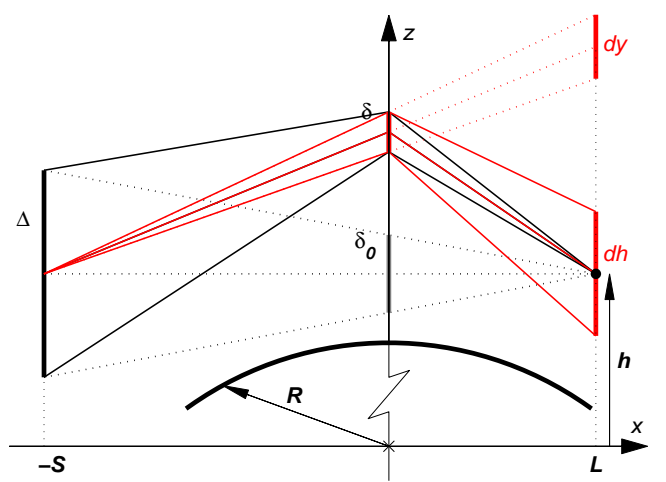

Figure 2. Simplified representation of the refraction plane geometry for the occultation of an extended light source at distance $S$ of the Earth's centre, observed by a satellite radiometer at distance $L$ from the limb.

pointing and stability knowledge has to meet the same requirements. We have the simple relations

$\beta^{\prime}(z)=\beta(z)+\alpha\left(z^{\prime}\right)$,

$z^{\prime}=z+S \beta$

$h=z^{\prime}+L \beta^{\prime}$.

Depending on the true altitude dependence of the refractivity profile $v\left(z^{\prime}\right)$, the system of Eqs. (12), (13), and (14) does not possess a general analytical solution and must be solved iteratively (an "exact" ray tracing solution would lead to the numerical solution of a boundary value problem for the differential Eq. 1). However, it is reasonable to linearize the profile of refracted angles around $z^{\prime}$ as

$\alpha\left(z^{\prime}\right) \simeq \alpha_{0}+\alpha_{1} z^{\prime}$,

where $\alpha_{0} \leq 0$ and $\alpha_{1} \geq 0$ for an exponentially decreasing density profile. By elementary algebra, one obtains the correct slope $\beta(z)$ under which the Sun ray is emitted to hit the sensor:

$\beta=\frac{h-\left(1+\alpha_{1} L\right) z-L \alpha_{0}}{L+S+L S \alpha_{1}}$.

If there was no refraction, the solar disc would span a vertical domain $\delta_{0}$ (of about $28 \mathrm{~km}$ ) at $x=0$ :

$\delta_{0}=\frac{L \Delta}{L+S}$.

By calculating the $\beta$ angles for the upper and lower edges of the Sun (Eqs. 16 and 13), we obtain the angular size of the refracted Sun:

$\delta=\frac{L \Delta}{L+S+L S \alpha_{1}}$.
We conclude that atmospheric refraction leads to two effects:

- The image of the light source is displaced by a positive elevation with respect to the unrefracted tangent altitude, leading to a non-negligible bias for the retrieval of the vertical concentration profile of any remotely sensed trace gas (see Table 1 below $40 \mathrm{~km}$ ). In particular, the centre of the Sun is shifted upward by

$$
z^{\prime}(h)-h=\frac{-L S\left(\alpha_{0}+\alpha_{1} h\right)}{L+S+L S \alpha_{1}} .
$$

- The atmosphere acts as a diverging lens and produces a smaller and real image of the Sun. The compression factor $D$ is obtained from Eqs. (17) and (18):

$$
D=\frac{\delta}{\delta_{0}}=\frac{L+S}{L+S+L S \alpha_{1}} .
$$

As observed from the sensor side and assuming for the moment a constant brightness of the source and the image, the radiometric signal will be proportional to the solid angle subtending the Sun image and hence reduced by the same factor $D$. This is a third effect of refraction, a decrease of the reference signal, that must be taken into account for the computation of atmospheric transmittance in occultation experiments. We call this effect "dilution" of the incoming irradiance according to the formalism presented in the following paragraph.

In some circumstances (typically when observing stars or planets), the angular size of the light source object is below the optical resolution and the concept of image flattening is useless. However the three refraction effects are still there, but it is easier to understand the dilution effect by reasoning on the refraction of a pencil of rays emitted from the Sun's surface and passing through the position of the refracted image at $x=0$ (see Fig. 2). In the absence of atmosphere, the radiative energy contained in this pencil would have hit the phase screen at $y$, illuminating an infinitesimal element $\mathrm{d} y$. Clearly, we obtain

$\frac{z^{\prime}-h}{S}=\frac{y-h}{L+S}$

and

$\mathrm{d} y=\frac{L+S}{S} \mathrm{~d} z^{\prime}$.

When atmospheric refraction is "switched on", the same pencil (hence the same amount of energy) will reach the detector at altitude $h$. When differentiating Eq. (14) for $z$ fixed $(\mathrm{d} z=0)$, we obtain

$\mathrm{d} h=\frac{\left(L+S+L S \alpha_{1}\right)}{S} \mathrm{~d} z^{\prime}$. 


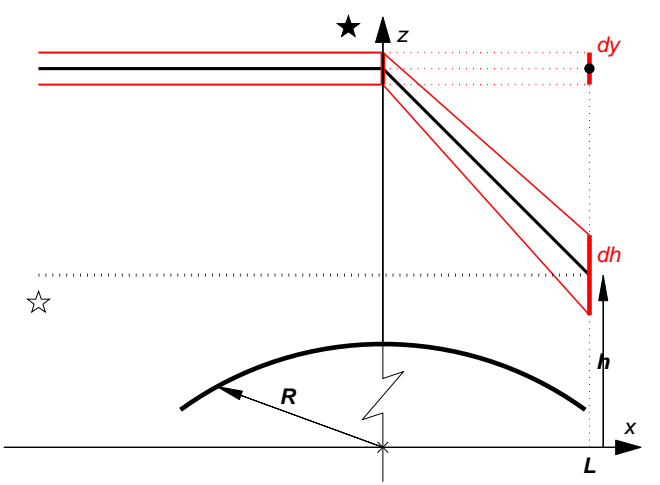

Figure 3. The refractive dilution mechanism for a pointlike distant source.

By conservation of energy, the observed irradiance is inversely proportional to the ratio of the infinitesimal elements on which the light is spread. It follows that

$\frac{\mathrm{d} y}{\mathrm{~d} h}=\frac{1}{1+\frac{L S \alpha_{1}}{L+S}}=D$,

which is the same result obtained in Eq. (20), from conservation of étendue (Chaves, 2008). A clear benefit of the dilution approach is to understand the decrease of the signal when observing a pointlike source as a star for which no image is measurable $\left(\frac{\Delta}{S} \simeq 0\right.$ ). In that case (see Fig. 3), we still observe two refractive effects: the tangent altitude change $(b \geq h)$ and the radiative dilution $(D \leq 1)$. For star, planetary and GSS occultations in the phase screen approximation, we simply obtain

$h=b+\alpha(b) L$,

$D=\frac{1}{1+L \alpha_{1}}$.

Even if the quantity $\alpha_{1}$ is small, one has to notice the amplification effect by the distance to the limb $L$ and there is no difficulty to alter the last equation for the general cases of $\alpha_{1}=\alpha_{1}(b)$ and $D=D(b)$ :

$D(b)=\frac{1}{1+L \frac{\mathrm{d} \alpha}{\mathrm{d} b}}$.

We want to emphasize here that the usual measurement of refraction by measuring the angular displacement from $h$ to $b$ (for pointlike source) or the image flattening (Gordley et al., 2009) can be equivalently replaced by a transmittance measurement, i.e. $D(b)$, taking into account other potential extinction mechanisms. Indeed, Eq. (27) may be re-written to obtain the following differential equation:

$\frac{\mathrm{d} \alpha}{\mathrm{d} b}=\frac{1}{L}\left(\frac{1}{D(b)}-1\right)$.
It is therefore possible to integrate Eq. (28) with boundary condition $\alpha(\infty)=0$ to retrieve the profile of refracted angles. A small difficulty subsists because $b$ is a priori unknown as it is determined by $\alpha$ itself. However, Eq. (25) is easily invertible and we also obtain

$D(h)=\frac{\mathrm{d} b}{\mathrm{~d} h}=1-L \frac{\mathrm{d} \alpha}{\mathrm{d} h}$

and the corresponding differential equation with the unrefracted tangent altitude as the independent variable:

$\frac{\mathrm{d} \alpha}{\mathrm{d} h}=\frac{1}{L}(1-D(h))$.

Once the refraction angle has been integrated as a function of the tangent altitude $h$, the refractive index profile is obtained by inverse Abel transform (Eq. 5) and the atmospheric density (and the associated error bar) can be easily approximated by using Eqs. (9) and (6).

\section{Application to star occultations by the GOMOS instrument}

\subsection{GOMOS instruments}

The GOMOS stellar occultation instrument has been extensively described elsewhere (e.g. Bertaux et al., 2010). Shortly, GOMOS was embarked on board the ENVISAT platform launched in 2002 in a heliosynchronous circular orbit at an altitude of $800 \mathrm{~km}$. After 10 years of operation, more than 860000 measurements have been carried out. GOMOS is a UV-visible-NIR spectrometer aimed at monitoring the vertical concentration profiles of minor atmospheric constituents such as $\mathrm{O}_{3}$ or $\mathrm{NO}_{2}$. Each spectral transmittance is obtained by dividing the measurements made through the atmosphere by the reference one performed outside the atmosphere. It is also equipped with an accurate pointing system required to track efficiently the setting star during the occultation. This mechanism consists of a plane mirror controlled by a steering front assembly (SFA) and driven by a fast Star Acquisition and Tracking Unit (SATU).

The GOMOS measurements are sensitive to atmospheric scintillations caused by atmospheric turbulence. To demodulate the amplitude fluctuations, two fast photometers have been added to measure simultaneously the star intensities in two spectral channels centred in the blue $(500 \mathrm{~nm})$ and in the red $(672 \mathrm{~nm})$. It turned out that this correction is almost perfect when the star sets along the orbital plane but does not work properly for very oblique occultations due to the presence of residual scintillation caused by horizontal turbulent structures. Photometer data have also been used to investigate atmospheric turbulence (Kan et al., 2014) and to derive high-resolution temperature profiles (Dalaudier et al., 2006) from the time delay induced by chromatic refraction. Here we focus on the low-frequency part of the refractive effects 

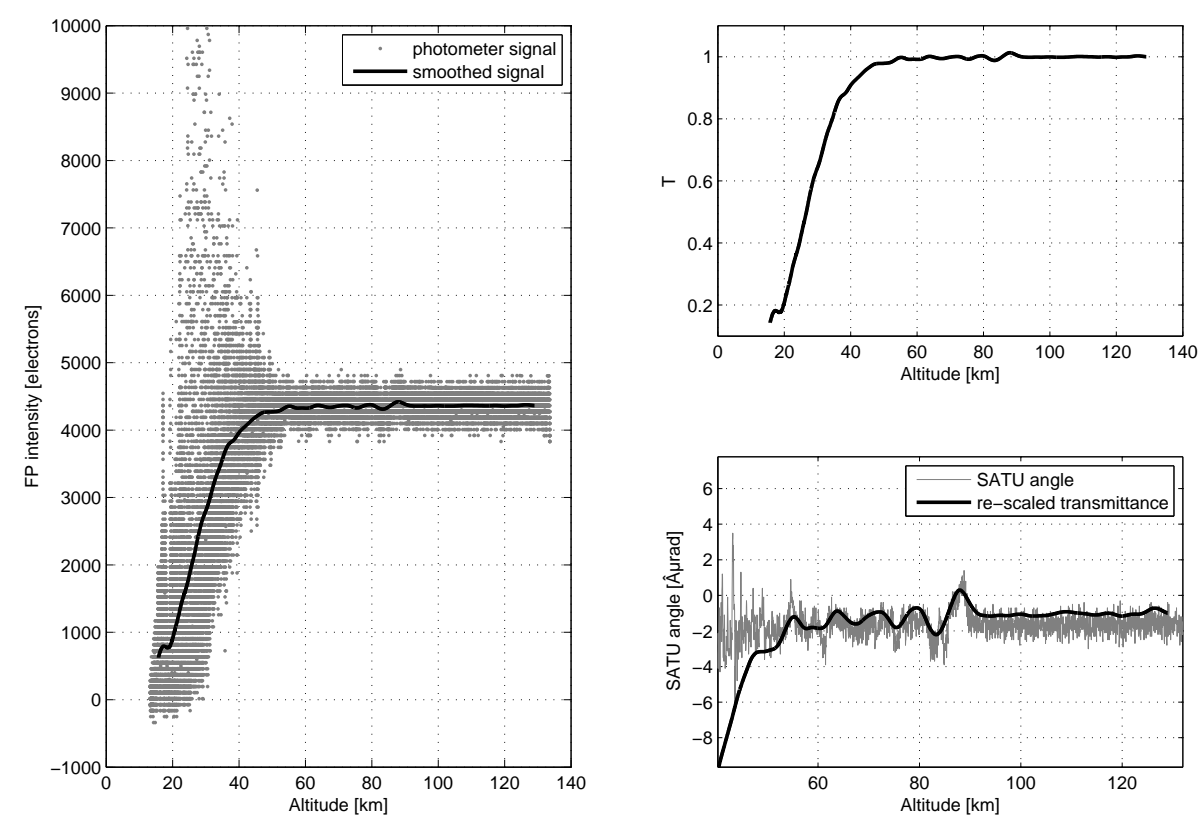

Figure 4. Left panel: raw and smoothed signals measured by the red GOMOS photometer during a full occultation. Right upper panel: associated transmittance profile. Right lower panel: SATU angle and re-scaled transmittance.

by showing how to apply the ARID algorithm to the photometer data for the retrieval of refractive angle profiles.

\subsection{Processing of GOMOS photometer data}

During GOMOS occultations, a part of the incoming star light is routed toward the fast photometers. They measure the stellar intensities with a high sampling frequency $(1 \mathrm{kHz})$ in limited wavelength bandwidths $(50 \mathrm{~nm})$. During one spectrometer acquisition $(0.5 \mathrm{~s})$, the photometers record 500 stellar intensity values, from which we extract a transmittance profile $T(h)$ as

$T(h)=\frac{I_{\mathrm{S}}(h)}{I_{\mathrm{r}}}$,

where $I_{\mathrm{S}}(h)$ is the signal of the photometer smoothed with a Hanning filter to remove the fluctuations due to atmospheric scintillation and $I_{\mathrm{r}}$ is the exo-atmospheric reference signal of the star computed from the median of all measurements with a tangent altitude greater than $105 \mathrm{~km}$. The left panel of Fig. 4 shows a typical intensity signal measured by the red photometer, together with its smoothed version. One can notice some oscillations (which are of instrumental nature due to intra-pixel sensitivity coupled with a too sharp star image focusing) observed at higher tangent altitudes (above $50 \mathrm{~km}$ ) that are strongly correlated with the measurements of the SATU angle needed to keep the image of the setting star in the centre of the CCD detectors.

In the following example, we have used only the measurements of the red photometers to minimize the impact of optical extinction due to Rayleigh scattering, and ozone and ni- trogen dioxide absorptions. The $\mathrm{NO}_{2}$ absorption slant path optical thickness $\delta_{\mathrm{NO}_{2}}$ at $30 \mathrm{~km}$ in the red channel is about $\delta_{\mathrm{NO}_{2}}(672 \mathrm{~nm}) \simeq 1.2 \times 10^{-3}$ and will be neglected hereafter.

Therefore, the transmittance $T_{\mathrm{m}}(h)$ deduced from the red photometer measurement at each tangent altitude $h$ can be expressed simply as

$T_{\mathrm{m}}(h)=D(h) T_{\mathrm{R}}(h) T_{\mathrm{O}_{3}}(h)$,

where $D$ represents the contribution of the refractive dilution, $T_{\mathrm{R}}$ the Rayleigh scattering and $T_{\mathrm{O}_{3}}$ the ozone absorption (retrieved in the GOMOS data processing). From Eq. (30) we obtain the following expression:

$\frac{\mathrm{d} \alpha}{\mathrm{d} h}=\frac{1}{L}\left(1-\frac{T_{\mathrm{m}}(h)}{T_{\mathrm{R}}(h) T_{\mathrm{O}_{3}}(h)}\right)$.

The transmittance $T_{\mathrm{R}}$ can be expressed as a function of the air scattering cross section $\sigma_{\mathrm{R}}$, the effective slant path length through the atmosphere $L_{\mathrm{R}}$ (i.e. the equivalent optical length over which the optical thickness reaches its exact value, see Eq. 37 hereafter) and the air number density $\rho$ :

$T_{\mathrm{R}}(h)=\exp \left(-\sigma_{\mathrm{R}} \rho(h) L_{\mathrm{R}}\right)$.

The following expression for $\sigma_{\mathrm{R}}$ (Bodhaine et al., 1999) has been used in this study:

$\sigma_{\mathrm{R}}=\frac{24 \pi^{3}}{\lambda^{4} \rho_{0}^{2}}\left(\frac{n(\lambda)^{2}-1}{n(\lambda)^{2}+2}\right)^{2} F_{\mathrm{K}}$, 


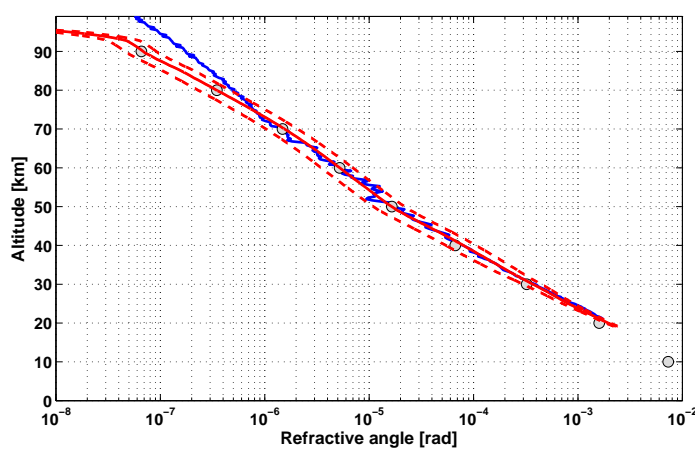

Figure 5. Refraction angle profiles retrieved using a single GOMOS occultation (star $\beta$ Hyi, 22 July 2002, 10:21:53 GMT, lat: $1^{\circ} \mathrm{S}$, long: $176^{\circ} \mathrm{E}$ ) from the red photometer (red thick line) with estimated errors due to photometer signals derived from Monte Carlo simulation (red dashed lines), from the GOMOS pointing measurement (in blue; notice that the GOMOS requirement for pointing stability was only "better than $40 \mu \mathrm{rad}$ " ) and compared with the analytical approximation (full circles) computed from Eq. (9).

in which $\lambda$ is the wavelength, $\rho_{0}=2.547 .10^{19} \mathrm{~cm}^{-3}$ is the air number density at standard temperature and pressure $\left(T_{0}=\right.$ $\left.288.15 \mathrm{~K}, P_{0}=1013.25 \mathrm{mb}\right), F_{\mathrm{K}}$ the King factor with a constant value of 1.06 (Lenoble, 1993) and $n$ the air refractive index. The number density $\rho(h)$ that appears in Eq. (34) can be expressed as a function of the refractivity using Eq. (6) and hence as a function of the refraction angle $\alpha$ (Eq. 9). Finally, we obtain

$\rho(h)=\frac{\rho_{0}}{C(\lambda)}|\alpha(h)| \sqrt{\frac{H_{\mathrm{t}}}{2 \pi\left(r_{\mathrm{t}}+h\right)}}$.

The effective length $L_{\mathrm{R}}$ of an exponentially decreasing atmosphere can be well approximated by

$L_{\mathrm{R}} \simeq \sqrt{2 \pi R_{\mathrm{t}} H_{\mathrm{t}}}$.

Finally, using Eqs. (34), (36) and (37) in Eq. (33), we obtain

$\frac{\mathrm{d} \alpha}{\mathrm{d} h}=\frac{1}{L}\left(1-\frac{T_{\mathrm{m}}(h)}{T_{\mathrm{O}_{3}}(h)} \exp \left(\frac{\sigma_{\mathrm{R}} \rho_{0}}{C(\lambda)} \alpha(h) H_{\mathrm{t}} \sqrt{\frac{R_{\mathrm{t}}}{R_{\mathrm{t}}+h}}\right)\right)$.

By numerically integrating this equation, we obtain $\alpha(h)$. One can notice that the scale height $H_{\mathrm{t}}$ is dependent on the temperature. In a first iteration, we have used climatological profiles of the scale height to obtain a first evaluation of the refraction angle $\alpha_{0}$ and of the associated number density and scale height. Then, an additional iteration was performed from these values to verify the convergence of the solution of Eq. (38). In all performed test cases, we observed that the method used is stable and provides profiles of refraction angles that are not dependent on the first-guess scale height.

\subsection{Results and comparisons}

Figure 5 shows the refractive angle derived by the ARID method from the GOMOS red photometer (in red) using measurements from one single occultation observed in July 2002. This vertical occultation (close to the orbital plane) was performed in full dark illumination condition (night). We have also compared the ARID numerical results obtained with the analytical approximation of Eq. (9), showing a fair agreement in the upper atmosphere. Typically, the relative differences decrease with the altitude from almost $20 \%$ at $20 \mathrm{~km}$ to a few percent at $90 \mathrm{~km}$.

An alternative way to obtain the refraction angles from a GOMOS occultation is to use the pointing angle of the tracking system. Indeed, at the beginning of the measurement, the main mirror is oriented in elevation and in azimuth toward the target star. Then, during the occultation, this orientation is modified to keep the star image in the centres of the detectors. In the GOMOS data, the elevation and azimuth angles are provided as a function of time. For a vertical occultation, the changes in elevation angle are due to the movement of the satellite on its orbit and to the refractive effects. Thus, the refraction angle is simply deduced by subtracting the geometric contribution from the sum of the elevation and SATU angles. Figure 5 also shows the refraction angle deduced from the pointing system, in satisfactory agreement with the values retrieved by using the ARID method and the analytical approximation.

\section{Application to solar occultations by the ORA instrument}

\subsection{Refractive dilution of the Sun}

In solar occultations from a LEO satellite without any imaging system, the observed transmittance is integrated over the angular size of the Sun, resulting in an apparent loss of vertical resolution for the retrieved vertical refractivity profile. However, this loss is apparent because the sampling rate of measurements is usually large with a strong overlap of the observed solar discs between two successive acquisitions that causes redundance. This redundance does not lead to an illconditioned inverse problem because the very large $S / N$ ratio is not limited by the brightness of the source but only by the dynamical scale of the sensor. Still, the inverse problem consists of retrieving a profile of refracted angles from a signal produced by a double integration: one along the optical path and one along the angular range under which the Sun is observed. Assuming the spherical homogeneity of the atmosphere around the geolocation of the measurement (this should be checked with respect to Eq. 10), we can make use of the symmetry of the problem (see Fig. 6). Hereafter we will consider the near-infrared domain where contributions of $\mathrm{O}_{3}$ absorption and Rayleigh scattering may be neglected. 
Table 2. Table of solar limb darkening parameters valid for $0.422<$ $\lambda[\mu \mathrm{m}]<1.1$.

$$
\begin{array}{ll}
a_{0} & 0.75267-\frac{0.265577}{\lambda} \\
a_{1} & 0.93874+\frac{0.265577}{\lambda}-\frac{0.004095}{\lambda^{5}} \\
a_{2} & -1.89287+\frac{0.012582}{\lambda^{5}} \\
a_{3} & 2.4223-\frac{0.017117}{\lambda^{5}} \\
a_{4} & -1.71150+\frac{0.011977}{\lambda^{5}} \\
a_{5} & 0.49062-\frac{0.003347}{\lambda^{5}} \\
\hline
\end{array}
$$

We consider that the Sun consists of horizontal slices spanning an angular domain described by the angle $\theta \in\left[\theta_{\mathrm{b}}, \theta_{\mathrm{t}}\right]$, where $\theta_{\mathrm{b}}=-\arctan \left(\frac{\Delta}{2 S}\right)$ and $\theta_{\mathrm{t}}=-\theta_{\mathrm{b}}$ respectively refer to the bottom and top edges of the Sun (Fig. 6). Each slice is seen with the same refraction geometry: the rays are grazing the same spherical surface at the unrefracted tangent altitude $h_{\theta}$ located at a satellite-limb distance $L_{\theta}$, whereas $L$ and $h$ refer to the Sun centre.

The relation between these quantities is obtained by a rotation matrix as

$L_{\theta}=L \cos \theta+h \sin \theta \quad h_{\theta}=-L \sin \theta+h \cos \theta$.

We can express the total measured transmittance $T(h)$ as

$T(h)=\int_{\theta_{b}}^{\theta_{\mathrm{t}}} G(\theta)\left(1-L_{\theta} \frac{\mathrm{d} \alpha_{\theta}}{\mathrm{d} h_{\theta}}\right) d \theta$,

where $G(\theta)$ is a normalized brightness distribution related to the solar limb darkening and

$\int_{\theta_{b}}^{\theta_{\mathrm{t}}} G(\theta) d \theta=1$

The apparent brightness of Sun is not homogeneous because radiation is emitted at different altitudes (i.e. different temperatures) in the photosphere and is attenuated through different layers. In this article, we will use the limb darkening parameterization of Neckel, as done by Dekemper et al. (2013). The relative intensity of a slice subtended by angle $\theta$ (with respect to the intensity of the same slice if it would have a constant brightness of 1) can be obtained by integrating the solar limb darkening curve along the slice of constant $\theta$.

The latitudinal dependence of the limb darkening parameterization $\operatorname{sld}(\beta)$ is expressed as a function of the cosine of the emission angle $\beta$ from the Sun surface by

$$
\begin{aligned}
\operatorname{sld}(\beta)= & a_{0}+a_{1} \cos (\beta)+a_{2} \cos (\beta)^{2}+a_{3} \cos (\beta)^{3} \\
& +a_{4} \cos (\beta)^{4}+a_{5} \cos (\beta)^{5},
\end{aligned}
$$

where the $a_{i}$ and their wavelength dependence are given in Table 2. After integration across the solar disc along the hor-

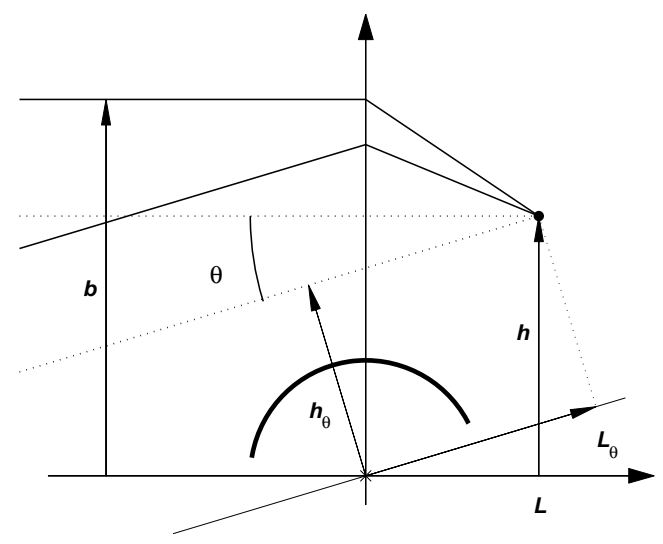

Figure 6. Geometry for angular integration across the solar disc.

izontal direction, the brightness distribution is obtained as

$$
\begin{aligned}
G(\theta)= & 8 \zeta\left(a_{0}+\frac{\pi a_{1}}{2 \Delta} \zeta+\frac{8 a_{2}}{3 \Delta^{2}} \zeta^{2}+\frac{3 \pi a_{3}}{2 \Delta^{3}} \zeta^{3}\right. \\
& \left.+\frac{128 a_{4}}{15 \Delta^{4}} \zeta^{4}+\frac{5 \pi a_{5}}{\Delta^{5}} \zeta^{5}\right) /\left(\pi \Delta^{2} G_{\lambda}\right)
\end{aligned}
$$

with

$\zeta(\theta)=\sqrt{\frac{\Delta^{2}}{4}-S^{2} \tan ^{2}(\theta)}$

and

$G_{\lambda}=a_{0}+\frac{2}{3} a_{1}+\frac{1}{2} a_{2}+\frac{2}{5} a_{3}+\frac{1}{3} a_{4}+\frac{2}{7} a_{5}$.

For the inversion, it is useful to work with the complementary transmittance $T_{\mathrm{c}}(h)=1-T(h)$. The retrieval of $f(\theta ; h)=\frac{\mathrm{d} \alpha_{\theta}}{\mathrm{d} h_{\theta}}$ is obtained from a set of $m$ complementary transmittance measurements $T_{\mathrm{c}}\left(h_{i}\right)$, recorded at successive nominal tangent altitudes $h_{i}$ :

$T_{\mathrm{c}}\left(h_{i}\right)=\int_{\theta_{b}}^{\theta_{\mathrm{t}}} G(\theta) L\left(\theta ; h_{i}\right) f\left(\theta ; h_{i}\right) d \theta \quad\{i=1 \ldots m\}$.

\subsection{Application to ORA data}

The ORA instrument, launched onboard the EUropean REtrievable CArrier (EURECA) in July 1992, had the unique opportunity to observe the relaxation of the Mount Pinatubo stratospheric aerosols, with a measurement coverage in the latitude range $\left[40^{\circ} \mathrm{S}-40^{\circ} \mathrm{N}\right]$, imposed by the low-orbit inclination of the satellite $\left(28^{\circ}\right)$. The solar occultation experiment aimed at the atmospheric limb remote sounding of $\mathrm{O}_{3}, \mathrm{NO}_{2}$, $\mathrm{H}_{2} \mathrm{O}$ and aerosol extinction vertical profiles in the UV to near IR range by using independent radiometric modules (Arijs et al., 1995).

ORA consisted of eight radiometers of similar design with broadband filters centred at 259, 340, 385, 435, 442, 600, 

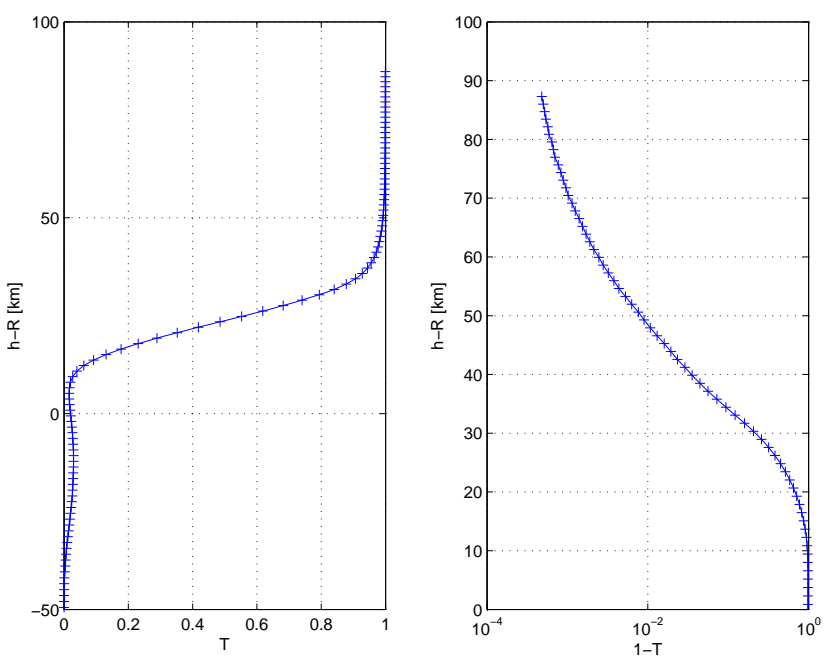

Figure 7. A typical transmittance measurement in a solar occultation observed by ORA. Notice the strong absorption by the Pinatubo stratospheric aerosol around $h-R \simeq 10 \mathrm{~km}$. The signal is produced by a current measurement and is virtually free of shot noise.

943, $1013 \mathrm{~nm}$, each containing a quartz window, an interference filter, and a simple optics followed by a photodiode detector. From August 1992 to May 1993 the instrument measured about 7000 sunrises and sunsets from its quasi-circular orbit at an altitude of $508 \mathrm{~km}$. The near-infrared channel at $1013 \mathrm{~nm}$ was mainly dedicated to stratospheric aerosol observations as it is very weakly affected by Rayleigh scattering (see Fig. 7).

The ORA data set has been unfortunately archived with a restricted altitude range and sampling, sufficient for an accurate retrieval of vertical profiles of ozone concentration and of stratospheric aerosol extinction. However, above 50 $60 \mathrm{~km}$, a contamination by stray light was clearly caused by the very large field of view of the instrument $\left( \pm 2^{\circ}\right)$. The unavailability of high-altitude measurements to correctly assess the stray-light contribution forced us to use an empirical stray-light removal algorithm above $50 \mathrm{~km}$, which constrains the transmittance to behave as an exponential of a low-order polynomial in tangent altitude. This was possible because the smooth signal is produced by a photodiode current measurement and virtually free of shot noise.

The large vertical extension of the solar disc at the geolocation of the occultation caused a strong overlap between successive transmittance measurements. Each acquisition combines different Sun slices (corresponding to paraxial rays at different tangent altitudes with respect to the Sun centre). Therefore a first step was to build a Lagrange interpolation matrix to map the set of paraxial rays into a $1 \mathrm{~km}$ regular altitude grid. The discretization allows us to re-write Eq. (46) under matrix form:

$\vec{y}=K \overrightarrow{\boldsymbol{f}}$

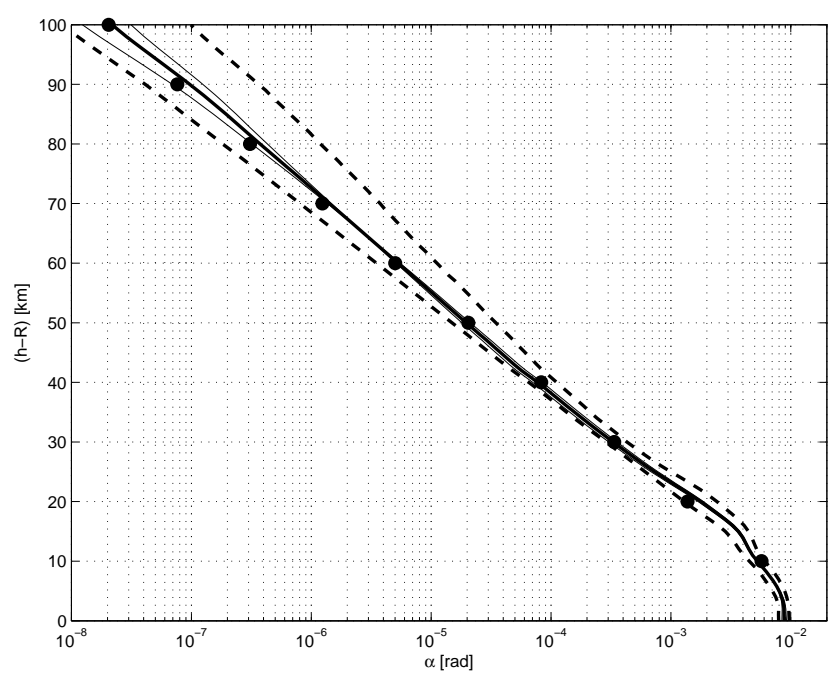

Figure 8. Median profile of atmospheric refraction angle obtained from the processing of 2836 ORA solar occultations observed between Aug 1992 and May 1993. Full thick line: median profile. Full dashed lines: $16 \%$ and $84 \%$ percentiles of the retrieved profiles distribution. Full thin lines: estimated errors due to signal digitization (16 bits). Full circles: refraction angles obtained by exact ray tracing for US76 standard conditions ( $\alpha_{b}$ in Table 1$)$.

where the forward model operator $K$ combines the brightness angular weights $G(\theta)$, the distances to the limb $L(\theta)$ and the unknown profile $f$ of refraction angles. As very little a priori information is known about the covariance matrix of the a priori profile, the standard application of a Bayesian optimal estimation method was not relevant for this case. Instead, after an appropriate scaling of all factors of Eq. (47), we implemented two numerical methods (Hansen, 1998): a classical Tikhonov regularization TIK (depending on a regularization parameter $\mu$ ) and a preconditioned conjugated gradient method PCG (iterated up to a maximal number of iterations $n_{\mathrm{i}}$ ). Two selection methods were also inter-compared to select $\mu$ or $n_{\mathrm{i}}$ : the classical Lcorner (LC) method searching for the accuracy-smoothing boundary (Hansen, 1998), and the Durbin-Watson (DW) method (Fussen, 1999; Durbin and Watson, 1950) that tries to minimize the correlation between the residuals. After several test cases and although both methods and regularization parameters give close results, the combination PCG-DW was selected because it turned out to be slightly more robust in perturbed cases.

Eventually, the retrieved $f(h)=\frac{\mathrm{d} \alpha}{\mathrm{d} h}$ was numerically integrated to obtain the vertical profile of refraction angles.

We have processed the full ORA data set (6821 occultations) from which we selected 2836 transmittance profiles that showed the least stray-light contamination. The statistical results are presented in Fig. 8. The median vertical profile of refraction angles is in good agreement with values obtained by ray tracing for a US76 atmosphere although the 
contamination by residual stray light is responsible for an important spread above $40-50 \mathrm{~km}$. The median agreement is about $5 \%$ in the $30-60 \mathrm{~km}$ range and $15 \%$ in the $60-100 \mathrm{~km}$ range. Below 20-30 km, the absence of cloud screening prevents an accurate assessment. With only 16 bits to code the signal, the digitization error was taken into account and is visible in the upper atmosphere.

It has to be underlined that the ARID method used in solar occultations can produce useful data over 6 orders of magnitude. The quality of the data is strongly related to the possibility of the stray-light removal, to the level of pointing stability during the acquisition of the reference radiance and during the occultation (typically $1 \mathrm{~min}$ ) and to the possibility of cloud screening at lower altitudes.

\section{Conclusions}

Geometrical optics is extensively used to describe the propagation of electromagnetic waves through an atmospheric medium. Many studies have been focused on the computation and the measurement of amplitude and phase fluctuations induced by a random medium, reflecting important properties of atmospheric turbulence. However, in this paper, we concentrated on the exploitation of the average refractive bending that is relevant to a ray tracing approach (neglecting diffraction). We demonstrate how atmospheric refraction is equivalently responsible for a change of the tangent altitude in limb remote sensing geometry and for a change (mostly an attenuation) of the apparent radiance of the light source. As occultation measurements are self-calibrating, it is then possible to process the refractive dilution curve to obtain the vertical profile of refraction angles. This is the basis of the ARID method that can be implemented in a very direct way for punctual sources like stars or planets by integration of a simple differential equation. As the numerical integration proceeds downward from the exo-atmospheric domain, the method is particularly well suited for upper atmospheric measurements to the limit of the radiometric sensitivity and the pointing stability. For extended sources like the Sun, a complementary angular inversion is necessary but it leads to a well-conditioned problem due to the very high signal-tonoise ratio.

We have applied ARID to GOMOS stellar and ORA solar occultation data with encouraging results. Our institute is presently designing a triple CubeSat PICASSO (PICosatellite for Atmospheric and Space Science Observations) that will host the spectral imager VISION (VIsible Spectral Imager for Occultation and Nightglow). This prototype instrument for atmospheric remote sensing from pico-satellites will be able to observe solar occultations in inertial mode thanks to its imaging capacity of the full solar disc. It should be able to measure refraction angles of about $0.5 \mu \mathrm{rad}$ at a tangent altitude of $80 \mathrm{~km}$. PICASSO has been very recently accepted and funded by ESA as an IOD (In Orbit Demonstration) mission.
Acknowledgements. This work has been partially funded by the PRODEX programme of the Belgian Scientific Policy Office (BELSPO) in support to the ORA and GOMOS experiences. PICASSO is presently funded by ESA under GSTP program "PICASSO Mission and VISION Miniaturized Hyperspectral Imager".

Edited by: C. von Savigny

\section{References}

Arijs, E., Nevejans, D., Fussen, D., Frederick, P., Ransbeek, E. V., Taylor, F. W., Calcutt, S. B., Werrett, S. T., Heppelwhite, C. L., Pritchard, T. M., Burchell, I., and Rodgers, C. D.: The ORA Occultation Radiometer on EURECA, Adv. Space Res., 16, 833836, 1995.

Bertaux, J. L., Kyrölä, E., Fussen, D., Hauchecorne, A., Dalaudier, F., Sofieva, V., Tamminen, J., Vanhellemont, F., Fanton d'Andon, O., Barrot, G., Mangin, A., Blanot, L., Lebrun, J. C., Pérot, K., Fehr, T., Saavedra, L., Leppelmeier, G. W., and Fraisse, R.: Global ozone monitoring by occultation of stars: an overview of GOMOS measurements on ENVISAT, Atmos. Chem. Phys., 10, 12091-12148, doi:10.5194/acp-1012091-2010, 2010.

Bodhaine, B. A., Wood, N. B., Dutton, E. G., and Slusser, J. R.: On rayleigh optical depth calculations, J. Atmos. Ocean. Tech., 16, 1854-1861, 1999.

Born, M. and Wolf, E.: Principles of Optics, 6th edn., Imprint, Oxford Pergamon Press, New York, USA, 1975.

Bracewell, R.: The Fourier Transform and its Apllications, McGraw-Hill, New York, USA, 1965.

Chaves, J.: Introduction to Nonimaging Optics, CRC Press, Boca Raton, FL, USA, 2008.

Dalaudier, F., Sofieva, V., Hauchecorne, A., Kyrölä, E., Laurent, L., Marielle, G. Retscher, C., and Zehner, C.: High resolution density and temperature profiling in the stratosphere using bichromatic scintillation measurements by GOMOS, in: Proceedings of the First Atmospheric Science Conference, European Space Agency, 2006, edited by: Lacoste, H. and Ouwehand, L., ESA SP-628, published on CDROM, pp. 34.1., 2006.

Dekemper, E., Vanhellemont, F., Mateshvili, N., Franssens, G., Pieroux, D., Bingen, C., Robert, C., and Fussen, D.: Zernike polynomials applied to apparent solar disk flattening for pressure profile retrievals, Atmos. Meas. Tech., 6, 823-835, doi:10.5194/amt-6823-2013, 2013.

Durbin, J. and Watson, G.: Testing for serial correlation in leastsquares regression, Biometrika, 37, 409-428, 1950.

Edlen, B.: The refractive index of air, Metrologia, 2, 71-80, 1966.

Elliot, J. L. and Olkin, C. B.: Probing planetary atmospheres with stellar occultations, Annu. Rev. Earth Pl. Sc., 24, 89-123, 1996.

Fussen, D.: An efficient algorithm for the large-scale smoothing of scattered data retrieved from remote sounding experiments, Ann. Geophys., 21, 1645-1652, doi:10.5194/angeo-211645-2003, 2003.

Gordley, L., Burton, J., Marshall, B. T., McHugh, M., Deaver, L., Nelsen, J., Russell, J. M., and Bailey, S.: High precision refraction measurements by solar imaging during occultation: results from SOFIE, Appl. Optics, 48, 4814-4825, doi:10.1364/AO.48.004814, 2009. 
Hajj, G. A., Kursinski, E. R., Romans, L. J., Bertiger, W. I., and Leroy, S. S.: A technical description of atmospheric sounding by GPS occultation, J. Atmos. Sol.-Terr. Phy., 64, 451-469, 2002.

Hansen, P. C.: Rank-deficient and discrete ill-posed problems: numerical aspects of linear inversion, Siam, 4, ISBN 0-89871-4036, 141-154, 1998.

Kan, V., Sofieva, V. F., and Dalaudier, F.: Variable anisotropy of small-scale stratospheric irregularities retrieved from stellar scintillation measurements by GOMOS/Envisat, Atmos. Meas. Tech., 7, 1861-1872, doi:10.5194/amt-7-1861-2014, 2014.
Lenoble, J.: Atmospheric Radiative Transfer, A. Deepak Pub., Hampton, VA, USA, 1993.

Miller, D., E.: Stratospheric attenuation in the near ultraviolet, P. R. Soc. A, 301, 57-75, 1967.

Sokolovskiy, S.: Inversions of radio occultation amplitude data, Radio Sci., 35, 97-105, 2000.

Wheelon, A. D.: Electromagnetic Scintillation, I. Geometrical Optics, Cambridge University Press, Cambridge, UK, 2001.

Wheelon, A. D.: Electromagnetic Scintillation, II. Weak Scattering, Cambridge University Press, Cambridge, UK, 2003. 